cases in groups 1 and in 3 cases in groups 2 , which required various types of anti-glaucoma operations. Cystic macular edema developed in one case 5 months after surgery as a result of uveitis exacerbation and was stopped by corticosteroid therapy.

Conclusion: Surgery of uveal cataracts in pts with SpA and other immuno-inflammatory diseases providing adequate pre- and postoperative anti-inflammatory therapy improves visual acuity and reduces the frequency of uveitis relapses. Monitoring of IOP is necessary in the postoperative period.

REFERENCES:

[1] Mora P., Gonzales S., Ghirardini S. et al. Perioperative prophylaxis to prevent recurrence following cataract surgery in uveitis patients a two-centre, prospective, randomized trial. Acta Ophthalmol. Scandinavica Fondation. 2016 Sept.; 94(6): e 390-394. http//doi. 10.1111/aos.12955. Epub 2016 Feb 5.

Disclosure of Interests: None declared

DOI: 10.1136/annrheumdis-2021-eular.1193

\section{POS0914 IS THERE AN ASSOCIATION BETWEEN AUTOANTIBODIES INDUCTION AND LOSS OF THERAPEUTIC EFFICACY IN PATIENTS WITH AXIAL SPONDYLOARTHRITIS AND PSORIATIC ARTHRITIS TREATED WITH ANTI-TNF- $\alpha$ AGENTS?}

A. Martins ${ }^{1}$, D. Santos Oliveira ${ }^{1}, 2$, F. R. Martins ${ }^{3}$, M. Rato ${ }^{1}$, F. Oliveira Pinheiro ${ }^{1}$ D. Fonseca ${ }^{4}$, B. M. Fernandes ${ }^{1}$, S. Garcia ${ }^{1}$, S. Pimenta ${ }^{1,5}$, M. Bernardes $^{1,5}$, L. Costa ${ }^{1}{ }^{1}$ Centro Hospitalar Universitário São João, Rheumatology, Porto, Portugal; ${ }^{2}$ Faculdade de Medicina da Universidade do Porto - FMUP, Center for Health Technology and Services Research (CINTESIS), Porto, Portugal; ${ }^{3}$ University Hospital Center of Algarve, Rheumatology, Faro, Portugal; ${ }^{4}$ Centro Hospitalar Vila Nova de Gaia / Espinho - Unit 1, Rheumatology, Vila Nova de Gaia, Portugal; ${ }^{5}$ Faculdade de Medicina da Universidade do Porto - FMUP, Rheumatology, Porto, Portugal

Background: Induction of autoantibodies is frequently observed in patients treated with a TNF- $\alpha$ blocker. According to other authors, the incidence of induction of antinuclear antibodies (ANA) and anti-double stranded DNA antibodies (anti-dsDNA) varies between $23-57 \%$ and $9-33 \%$, respectively. However, it is unknown whether the induction of these autoantibodies affects the pharmacokinetics and bioavailability of biotherapy and, consequently, reduces the efficacy and safety of the drug.

Objectives: To analyze if there is an association between autoantibodies induc tion and therapeutic efficacy in a monocentric cohort of patients with axial spondyloarthritis (axSpA) and psoriatic arthritis (PsA) treated with anti-TNF- $\alpha$ agents Methods: The authors performed a retrospective analysis of patients with axSpA and PsA treated in our University Hospital with a TNF- $\alpha$ blocker as first biologic agent, and analysed the autoantibodies induction rate after 12 (T12) and 24 (T24) months of therapy. Then, they investigated the influence of autoantibodies in therapeutic efficacy at T12 and T24. Clinical evaluation, laboratory findings including erythrocyte sedimentation rate (ESR) and C-reactive protein (CRP) and disease activity and functional scores (Bath Ankylosing Spondylitis Disease Activity Index - BASDAI, AS Disease Activity Score with CPR - ASDAS-CRP, Bath AS Functional Index - BASFI) were collected from reuma.pt and medica records. For PsA patients, Disease Activity Score-28-CRP (DAS28-CRP), Simple Disease Activity Index (SDAI), Clinical Disease Activity Index (CDAI) and Health Assessment Questionnaire (HAQ) scores were also collected. Patients with positive ANA (titer $>1 / 100$ ) prior to anti-TNF- $\alpha$ therapy were excluded. Continuous variables were analyzed using a t-test and categorical variables using a Chisquare test. $P$-value $<0.05$ was considered statistically significant.

Results: In the axSpA group, 235 patients were included, 44.5\% were females, mean age at diagnosis of $42.3 \pm 12.4$ years and median disease duration of 11.5 (IQR 6.0-21.0) years. Positive ANA were observed in $16.9 \%$ at T12 and $26.3 \%$ at T24 and positive anti-dsDNA in $3.4 \%$ at $\mathrm{T} 12$ and $3.8 \%$ at T24, with similar conversion rates between different anti-TNF drugs and no significant gender difference. A significant difference in ASDAS-CPR was found in axSpA patients with and without ANA at T12 ( $p=0.047)$. ASDAS-CPR was 1.16 times higher in patients with ANA comparing to patients without them. However, no difference was found in the others disease activity and functional scores at T12. Furthermore, no significant difference, including ASDAS-CPR, was found at T24. Also, there was no significant difference found when comparing patients with and without anti-dsDNA. In the PsA group, 94 patients were included, 46.8\% were females, mean age at diagnosis of $46.7 \pm 11.7$ years and median disease duration of 11.5 (IQR 6.5-16.5) years. Positive ANA were found in $14.9 \%$ at T12 and $21.3 \%$ at T24 and positive anti-dsDNA in $2.1 \%$ at T12 and $3.2 \%$ at T24. When comparing the groups with and without ANA and with and without anti-dsDNA at T12 and T24, no significant difference in disease activity and functional scores was found.

Conclusion: This study revealed high rates of serology conversion, similar to the rates described before. The authors found that ASDAS-CPR was higher in axSpA patients with ANA after 12 months of therapy. However, this difference was no longer evident after 24 months. No other significant difference was found between patients with and without ANA or with and without anti-dsDNA. The authors consider that the induction of autoantibodies may interfere with the response to anti-TNF- $\alpha$ therapy in a short and initial period of time. Long-term follow-up data are lacking to say whether that influence will disappear consistently over the long run, as they observed after 12 months of therapy. However, they can state that, a priori, seroconversion should not lead to treatment suspension because of concerns about loss of efficacy.

Disclosure of Interests: None declared

DOI: 10.1136/annrheumdis-2021-eular.1287

\section{POS0915 SUSTAINABILITY OF IXEKIZUMAB RESPONSE AT THE INDIVIDUAL PATIENT LEVEL OVER TIME IN RADIOGRAPHIC AXIAL SPONDYLOARTHRITIS}

S. Ramiro $^{1}$, R. Bolce ${ }^{2}$, D. Sandoval ${ }^{2}$, A. Kronbergs ${ }^{2}$, S. Y. Park ${ }^{2}$, B. Wu ${ }^{3}$, J. A. Walsh' ${ }^{4}{ }^{1}$ Leiden University Medical Center (LUMC), Department of Rheumatology, Leiden, Netherlands; ${ }^{2}$ Eli Lilly and Company, Lilly Corporate Center, Indianapolis, United States of America; ${ }^{3}$ IQVIA RDS Canada ULC, QC Canada, Kirkland, Canada; ${ }^{4}$ University of Utah, Division of Rheumatology and the Department of Dermatology, Salt Lake City, United States of America

Background: Ixekizumab (IXE) has demonstrated efficacy in treating the signs and symptoms of radiographic axial spondyloarthritis ( $r$-axSpA) in patients (pts) up to week (Wk) 52 [1]. Pts may experience fluctuations in the improvements of their symptoms, which may impact their overall quality of life [2]. Providing data at the pt level is important to support consistency and sustainability of efficacy. Objectives: To analyse IXE response for sustainability at the individual pt level between Wks 16 and 52 .

Methods: COAST-V (NCT02696785) and COAST-W (NCT02696798) were two phase 3, multicentre, randomised, double-blind, placebo-controlled studies (adalimumab was the active control in COAST-V). Pts with active r-axSpA who were either biologic disease modifying anti-rheumatic drug (bDMARD) naïve (COAST-V) or, inadequate responders or intolerant to up to 2 TNF inhibitors (COAST-W) received IXE every 4 weeks (Q4W) or every 2 weeks (Q2W). Only IXE Q4W pts were included in the present analysis (approved dosage for the indication). Separate analyses were conducted on pts who achieved either endpoint; ASAS40 or ASDAS $<2.1$. The proportion (\%) of pts who achieved either ASAS40 or ASDAS $<2.1$, at Wk 16 and at each visit out to Wk 52 (total of 8 visits) was assessed. Missing data were imputed as non-response. Heatmaps were used to depict the data analysed at the individual patient level across all visits Results: In COAST-V, 81 pts enrolled in the trial were initially randomised to IXE Q4W. Of those, 48\% ( $\mathrm{N}=39)$ of pts achieved ASAS40 at Wk 16 (Figure 1), of which $59 \%(\mathrm{~N}=23)$ maintained ASAS40 at every visit afterwards. In total, $85 \%(\mathrm{~N}=33)$ of the ASAS40 achievers at Wk 16 maintained ASAS40 with some $(26 \%(\mathrm{~N}=10))$ fluctuations, between ASAS40 and ASAS20. In COAST-W, 114 pts enrolled in the trial were initially randomised to IXE Q4W. Of those, $25.4 \%(\mathrm{~N}=29)$ pts achieved ASAS40 at Wk 16, of which, 69\% ( $\mathrm{N}=20)$ maintained ASAS40 at every visit afterwards. In total, $83 \%(\mathrm{~N}=24)$, of the ASAS40 achievers at $\mathrm{Wk} 16$ maintained ASAS40 with some $(14 \%(\mathrm{~N}=4))$ fluctuations, between ASAS40 and ASAS20. Of the 81 pts initially randomised to IXE Q4W in COAST-V, 35 pts achieved low disease activity (ASDAS $<2.1$, LDA) at Wk 16. Of those, $54 \%(\mathrm{~N}=19)$ of pts maintained LDA at every visit afterwards. In total, $91 \%(\mathrm{~N}=32)$ of the ASDAS-LDA

\section{ASAS40}

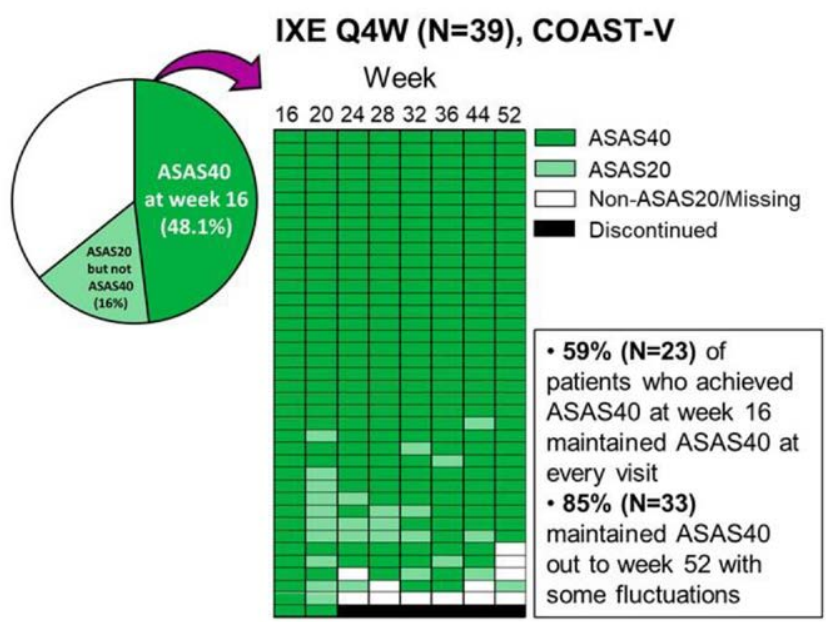

Figure 1. Heatmap diagram depicting the sustained effect of IXE over time at the pt level in pts with r-axSpA from COAST-V. Each row corresponds to an individual ASAS40 responder at Wk 16. 